\title{
A 10-bit 100 MSamples/s BiCMOS D/A Converter
}

\author{
Jørgensen, Ivan Harald Holger; Tunheim, Svein Anders
}

Published in:

Proc. 1996 IEEE International Symposium on Circuits and Systems

Link to article, DOI:

10.1109/ISCAS.1996.539975

Publication date:

1996

Document Version

Publisher's PDF, also known as Version of record

Link back to DTU Orbit

Citation (APA):

Jørgensen, I. H. H., \& Tunheim, S. A. (1996). A 10-bit 100 MSamples/s BiCMOS D/A Converter. In Proc. 1996 IEEE International Symposium on Circuits and Systems (pp. 425-428). IEEE.

https://doi.org/10.1109/ISCAS.1996.539975

\section{General rights}

Copyright and moral rights for the publications made accessible in the public portal are retained by the authors and/or other copyright owners and it is a condition of accessing publications that users recognise and abide by the legal requirements associated with these rights.

- Users may download and print one copy of any publication from the public portal for the purpose of private study or research.

- You may not further distribute the material or use it for any profit-making activity or commercial gain

- You may freely distribute the URL identifying the publication in the public portal

If you believe that this document breaches copyright please contact us providing details, and we will remove access to the work immediately and investigate your claim. 


\title{
A 10-BIT 100 MSAMPLES/S BICMOS D/A CONVERTER
}

\author{
Ivan Harald Holger Jørgensen ${ }^{1}$ \\ Svein Anders Tunheim ${ }^{2}$ \\ ${ }^{1}$ Electronics Institute, Building 349, \\ Technical University of Denmark (DTU), DK-2800 Lyngby, Denmark \\ Phone: (+45) 459342 07, Fax: (+45) 458801 17, E-mail: ihhj@ei.dtu.dk \\ ${ }^{2}$ SINTEF, P.O.Box 124 Blindern, N-0314 OSLO, Norway. \\ Phone: (+47) 220673 00, Fax: (+47) 220673 50, E-mail: Svein.Anders.Tunheim@si.sintef.no
}

\begin{abstract}
A 10-bit 100MSamples/s current-steering D/A converter (DAC) has been designed and processed in a $0.8 \mu \mathrm{m}$ BiCMOS process. The DAC is intended for applications using direct digital synthesis, and focus has been set on reducing dynamic nonlinearities to achieve a high spurious free dynamic range (SFDR ${ }^{1}$ ) at high generated frequencies. The main part of the DAC consists of a matrix of current cells. Each current cell contains an emitter-coupled logic (ECL) flip-flop, clocked by a global ECL clock to ensure accurate clocking. A bipolar differential pair, steered by the differential output of the ECL flip-flop, is used in each current cell to steer the current. The DAC operates at $5 \mathrm{~V}$, and has a power consumption of $650 \mathrm{~mW}$. The area of the chip-core is $2.2 \mathrm{~mm} \times 2.2 \mathrm{~mm}$. The measured integral nonlinearity (INL) and differential nonlinearity (DNL) were both approximately 2 LSB. At a generated frequency of $f_{g} \approx 0.3 \cdot f_{s}\left(f_{s}=100 \mathrm{MSam}\right.$ $p l e s / s$ ), the measured SFDR was approximately $43 \mathrm{~dB}$.
\end{abstract}

\section{INTRODUCTION}

High-speed and high resolution DACs are used in various applications such as direct digital synthesis (DDS), arbitrary waveform generation (AFG), new TV/Video systems and communications local oscillators. For these applications the dynamic performance of the DAC is very critical. For the DAC described in this paper, focus has been set on achieving a high spurious free dynamic range (SFDR) at high generated frequencies, $f_{g}$, compared to the conversion rate, $f_{s}$ (100MSamples $/ s$ ), i.e., $f_{g} \approx 0.3 \cdot f_{s}$. Any static or dynamic nonlinearity will generate spurious frequencies at the DAC output. For current steering DACs static nonlinearities can be reduced significantly by careful layout and by using different kinds of switching schemes for the current cells [5], [6]. The main dynamic nonlinearities are nonequal and code dependent rise and fall times, delay between rise and fall transients, and nonlinear clock and data feedthrough. For a typical current steering DAC one of the main challenges is to generate steering signals for the current cells with very little skew [1], [3], [5]. A solution is to use clocked current cells. One then has to generate a global clock for the current cells that has an acceptable low clock skew over the current cell matrix.

\footnotetext{
${ }^{1}$ SFDR is measured from the generated frequency to the highest harmonic or non-harmonic spur within the frequency band $0-f_{s} / 2$.
}

Clock-skew can more easily be controlled than the delays of different steering signals, therefore the use of clocked current cells is an advantage.

The DAC presented in this paper is constructed using this scheme. Each current cell contains an ECL flip-flop. The skew between the steering signals for the different current cells are then controlled by a global clock signal.

The DAC architecture is described in section II, and the current cells are described in section III. Section IV gives some considerations about the chip layout, and in section $\mathrm{V}$ the measurement results are presented.

\section{DAC ARCHITECTURE}

The DAC is based on the well-known current steering principle [1]-[7]. The DAC architecture is shown in Fig. 1.

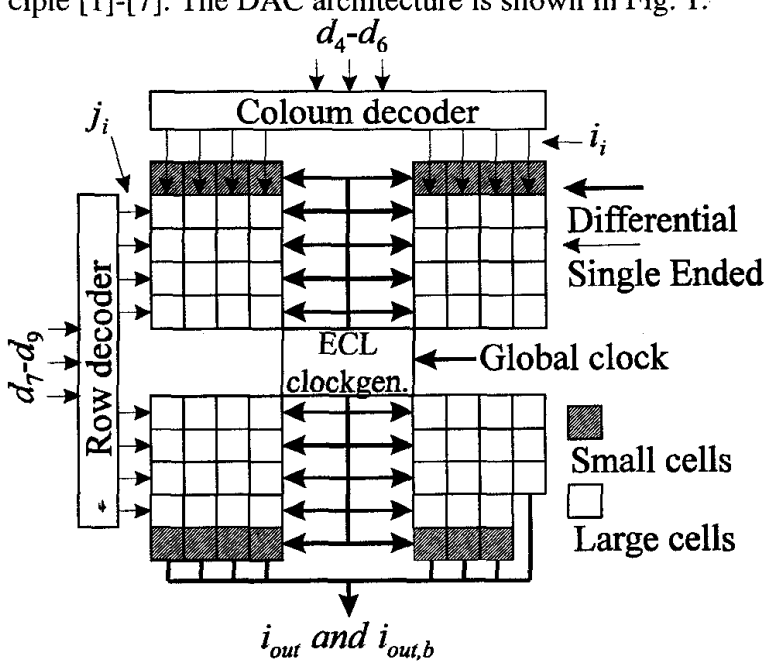

Fig. 1. DAC Architecture

The main part of the DAC consists of a matrix of current cells. The current cells are arranged in a matrix. This matrix consists of 63 large current cells that determine the 6 most significant bits (6 MSB), and 15 smaller cells that determine the 4 least significant bits (4 LSB). One large current cell sinks a current 16 times the current in a small current cell. The DAC operates as follows: The digital input word, $d_{9}(\mathrm{MSB})-d_{0}(\mathrm{LSB})$, is stored in an input register. The $6 \mathrm{MSB}$ 
are decoded into row, $j_{0}$ to $j_{7}$, and column, $i_{0}$ to $i_{8}$, signals. All signals (the $4 \mathrm{LSB}, i_{p}$ and $j_{q}$ ) are then latched before fed to the current cells. In Fig. 1 the registers and latches are contained in the column and row decoders. The decoding ensures that a minimum number of the large current cells are turned on or off; this reduces dynamic nonlinearities. The hierarchical switching scheme [6] is used for the large current cells to reduce nonlinearities. This switching scheme reduces both symmetrical and graded errors. For the small current cells, a simple "chessboard scheme" is used controlled by the digital signals $d_{3}-d_{0}$.

In each current cell (see Fig. 2) the CMOS steering signals, $i_{p}, i_{p+1}$ and $j_{q}$, are decoded by CMOS logic. The decoded signal is then converted into ECL levels and latched by an ECL flip-flop clocked by the global differential ECL clock. Thus, one reduces the problem with skew between the steering signals over the matrix. The differential current outputs of all current cells are connected to the DAC output pins $i_{\text {out }}$ and $i_{\text {out }, b}$ as shown in Fig. 1. These are, externally, each connected to a $50 \Omega$ resistor that again is connected to the positive power supply $(5 \mathrm{~V})$. The maximum output current from the DAC is designed to be $20 \mathrm{~mA}$. The ECL clock generator is placed in the middle of the current cell matrix to minimise clock skew. This also causes the clock skew to be a symmetrical error. Therefore it will be reduced by the hierarchical switching scheme.

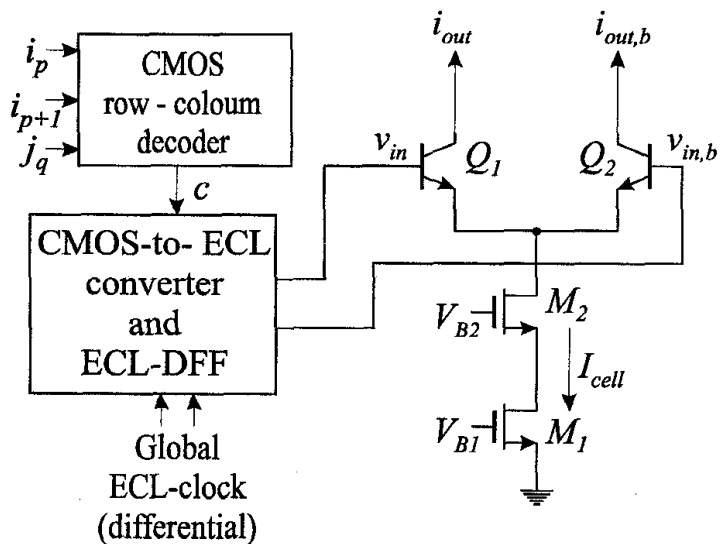

Fig. 2. The large current cell

\section{Current Cells}

In a previous design of a 100 MSamples/s 10 -bit DAC [1] the INL and DNL were found to be less than $1 \mathrm{LSB}$. The static nonlinearities for this DAC were acceptable whereas the dynamic performance at high generated frequencies compared to the sampling rate was very poor. Focus in the design of the DAC presented in this paper was therefore set on reducing dynamic nonlinearities to reduce the effects of these at high generated frequencies. This was done be designing very fast current cells.
A simplified schematic of the large current cells is shown in Fig. 2 [2]. A differential pair with bipolar transistors, $Q_{I}$ and $Q_{2}$, is used to steer the current. The cascode current sink consists of two NMOS transistors $M_{1}$ and $M_{2}$. The current cell includes a CMOS input section which decodes the row and column signals. The decoded signal is converted into a differential ECL signal that is latched before fed to the differential pair, $Q_{1}$ and $Q_{2}$.

The use of a bipolar differential pair offers several advantages compared to the MOS counterpart. As the DAC should operate at a very high conversion rate, it is obvious to use fast bipolar transistors to toggle the current $I_{\text {cell }}$ between the two outputs. Furthermore, the required voltage swing at the input, $v_{i n}-v_{i n, b}$, is much lower compared to the input swing of a MOS differential pair in a practical design. The differential input voltage which guaranties that the total erroneous output current from all $N_{c e l l s}$ current cells is less than $1 / 2 \mathrm{LSB}$ of the total current, is given by (1). In (1) the collector currents $I_{C 1}$ and $I_{C 2}$ are given by the well-known exponential equation for the bipolar transistor. $V_{T}$ is the thermal voltage which equals $26 \mathrm{mV}$ at room temperature.

$$
\frac{I_{C 1}}{I_{C 2}}>2^{N+1} N_{\text {cells }} \Rightarrow \Delta v_{i n}=v_{i n}-v_{i n, b}>V_{T} \ln \left(2^{N+1} N_{\text {cells }}\right)
$$

Using the number of bits $N=10, N_{\text {cells } s}=64$ (63 large, plus 15 small which equal approximately 1 large) we find that $\Delta v_{b e}>12 V_{T}=312 \mathrm{mV}$. This small ECL voltage swing and the fast ECL flip-flop output signals potentially give low dynamic nonlinearities at the DAC output.

The setup time of the output, $c$, from the CMOS input section in the current cell varies strongly depending on the input signals $i_{p}, i_{p+1}$ and $j_{q}$. If these CMOS signals, $c$, were used to steer the current cells directly the output signal from the DAC would contain higher levels of nonlinearities. The output from the CMOS part is therefore latched in the ECL section. The skew between the steering signals, $v_{i n}-v_{i n, b}$, is therefore only dependent on the clock skew in the global ECL clock. The worst case clock skew over the matrix is designed to be less than $8 p s$.

The small current cells have the same architecture as the large ones but $i_{p}$ is connected to $V_{d d}, i_{p+1}$ is connected to ground and the cells are only steered by $j_{q}$.

For the bipolar transistor the relation between the terminal currents is $I_{e}=I_{b}+I_{c}$ or $I_{e}=(1+\beta) \cdot I_{b}$. The current amplification factor, $\beta$, is process dependent and as bipolar transistors are used in the differential pair it is important to evaluate the influence of this in the collector current. We find:

$$
I_{c}=\alpha \cdot I_{e}, \quad \alpha=\frac{\beta}{1+\beta}
$$

The sensitivity of $\alpha$ with respect to $\beta$ :

$$
S_{\beta}^{\alpha}=\frac{\partial \alpha}{\partial \beta} \cdot \frac{\beta}{\alpha}=\frac{1}{(1+\beta)^{2}} \cdot \frac{\beta}{\alpha}
$$

The relative variation in $\alpha$ is approximately given by 


$$
\frac{\Delta \alpha}{\alpha} \approx S_{\beta}^{\alpha} \frac{\Delta \beta}{\beta}
$$

Assuming $\beta=120$ and $\Delta \beta / \beta$ better than $2 \%$ for transistors on the same chip, one obtain a $\Delta \alpha / \alpha$ of approximately $0.02 \%$. This will be of minor importance compared to other mismatch sources, such as ground potential variations and mismatch between NMOS current sinks (i.e., geometrical mismatch, threshold mismatch etc.).

\section{LAYOUT CONSIDERATIONS}

The DAC is processed in a $0.8 \mu \mathrm{m}$ double-metal double-poly BiCMOS process. To minimise switching noise from the CMOS- and ECL-logic to couple to the output of the DAC, the power supplies for the CMOS- and ECL-logic and the current sinks are routed separately. The current $I_{\text {cell }}$ from each large current cell introduces a voltage drop in the analogue ground. The analogue ground wires are therefore made wide. Also, several pads on each side of the current cell matrix are used to connect the analogue ground. Furthermore, all different parts of the circuitry are surrounded by guardrings and all major parts by double guardrings to minimise noise injected to the output through the substrate. A separate power pad has been used for the n-well parts of the guard rings. All bias lines, both to the ECL-logic and the current sinks in the current cells, have both local and global decoupling capacitors. Horizontal and vertical shielding between the analogue routing and digital data lines (both CMOS and ECL) have been used to minimise coupling between these. Careful layout and design were necessary to obtain sufficient time margin for the data setup with respect to the ECL clock. The chip was mounted in a 44 pin CLCC package.

Clock skew, ground potential variation, etc., that generate dynamic nonlinearities at the DAC output have been taken into account and were designed to introduce errors at the output, each less than $1 / 2$ LSB. The switching scheme will tend to cancel must of them. The total error at the output is therefore not the sum of all errors. The size of the chip-core is $2.2 \mathrm{~mm} \times 2.2 \mathrm{~mm}$.

\section{EXPERIMENTAL RESULTS}

The performance of a DAC is normally evaluated by considering the static and dynamic nonlinearities. The static nonlinearities INL and DNL are presented in Fig. 3 and Fig. 4. All measurements were performed with single ended output as no differences were seen between single ended and differential output.

The INL and DNL show that the current cells are not matched within an accuracy of 10 bit.

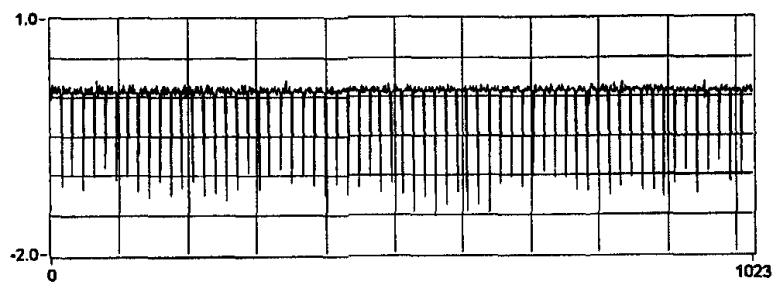

Fig. 3. Measured differential nonlinearity (DNL) versus input code.

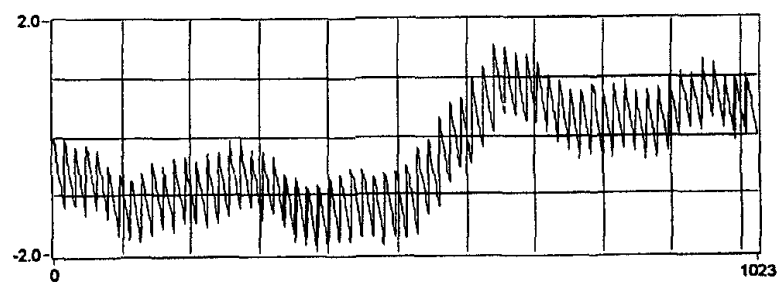

Fig. 4. Measured integral nonlinearity (INL) versus input code.

The dynamic performance of the DAC can still be evaluated separately. This is done by letting the DAC generate two different frequencies, one low and one high. When generating a low frequency the static nonlinearities will dominate and cause the spurious frequencies at the output. At high frequencies the dynamic nonlinearities will dominate and the effect of these can be viewed as the difference between the two spectra.

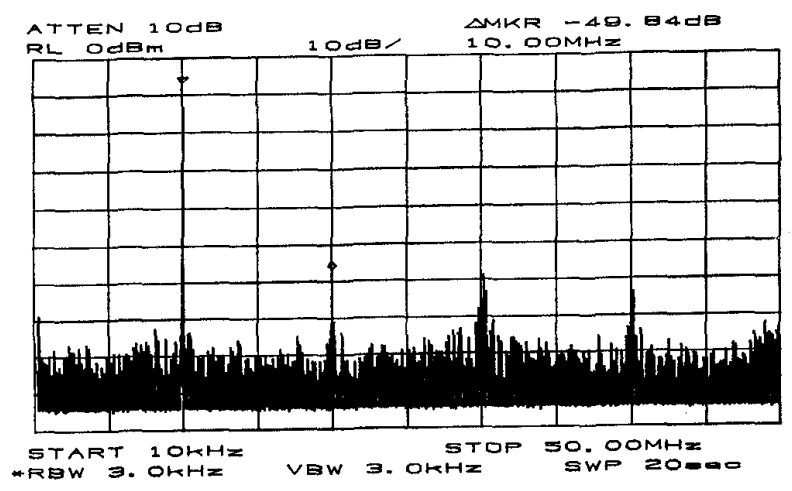

Fig. 5. The measured spectra for $f_{s}=100 \mathrm{MSamples} / \mathrm{s}$; $f_{g}=(820 / 8192) \cdot f_{s} \approx 0.1 \cdot f_{s}$.

The dynamic performance is evaluated by forcing a digital sine-wave to the input of the DAC. The digital sines are saved in a cyclic $8 k$-memory, i.e., 8192 samples with no delay in the cyclic readout. To ensure phase continuity the data are obtained by generating $f_{g}$ as $(n / 8192) \cdot f_{s}, n$ integer. A Colby Instruments Inc. SG8000A was used as the reference clock and an HP8563A spectrum analyser was used to 
measure the spectra which are presented in Fig 5 and Fig. 6 for $f_{g} \approx 0.1 \cdot f_{s}$ and $f_{g} \approx 0.3 \cdot f_{s}$. The result in Fig. 5 shows that the SFDR is approximately $50 \mathrm{~dB}$ at $f_{g} \approx 0.1 \cdot f_{s}$. The SFDR in Fig. 6 is further reduced by $7 \mathrm{~dB}$ to approximately $43 \mathrm{~dB}$.

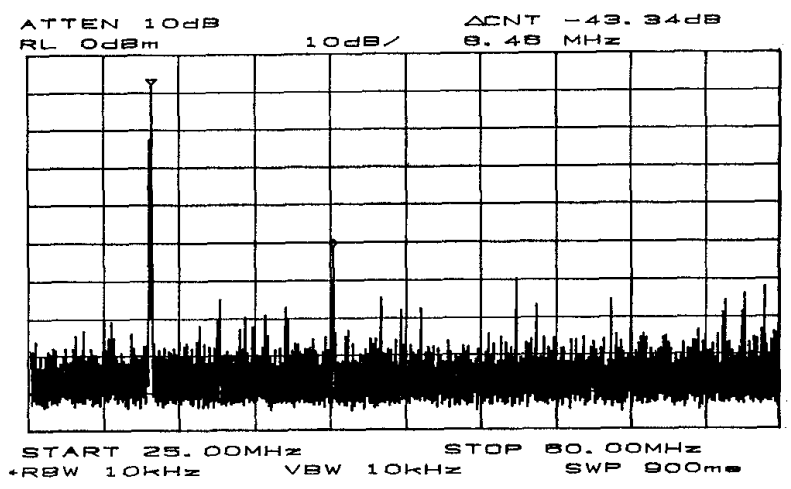

Fig. 6. The measured spectra for $f_{s}=100$ MSamples $/ s$; $f_{g}=(2501 / 8192) \cdot f_{s} \approx 0.3 \cdot f_{s}$.

For three different ratios between the generated frequency and sampling frequency the SFDR was measured for $10 \mathrm{MHz}<f_{\mathrm{s}}<150 \mathrm{MHz}$. The results are shown in Fig. 7 .

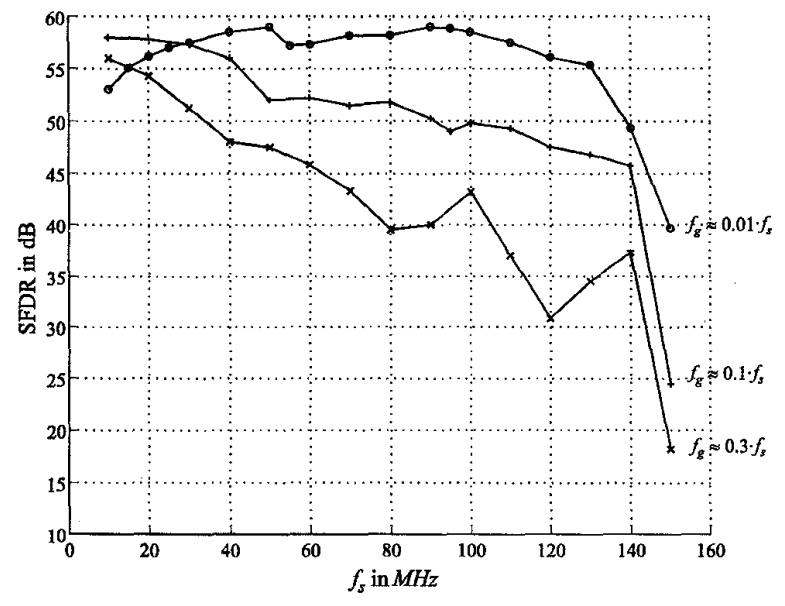

Fig. 7. The measured SFDR for $f_{g}=(79 / 8192) \cdot f_{s} \approx$ $0.01 \cdot f_{s}, f_{g} \approx 0.1 \cdot f_{s}$ and $f_{g} \approx 0.3 \cdot f_{s}$ as a function of $f_{s}$.

From Fig. 7 it can be seen that the DAC operated up to a sampling rate of 140MSamples/s after which the DAC did not generate a sine wave at the output. This was due to internal timing problems. The SFDR at $f_{s}=140 \mathrm{MSamples} / \mathrm{s}$ is approximately $6 d B$ lower than that of the test with $f_{s}=$ 100 MSamples/s. This corresponds well with the fact that the energy of one LSB is approximately halved and the dynamic nonlinearities are the same which result in a reduction in the SFDR of $6 \mathrm{~dB}$. The power consumption was $650 \mathrm{~mW}$. Be- cause the INL and DNL are larger than $1 \mathrm{LSB}$ one does not obtain a SFDR of $60 \mathrm{~dB}$ at low generated frequencies compared to the sampling frequency. For a constant ratio between $f_{g}$ and $f_{s}$ the SFDR increases when $f_{s}$ is decreased. This is again due to the fact that the dynamic nonlinearities become relatively less significant as the energy of one LSB is increased.

\section{Conclusion}

A 10-bit 100MSamples/s current steering DAC has been designed and fabricated in a $0.8 \mu \mathrm{m}$ BiCMOS process. The core of the DAC consists of a matrix of current cells. Each current cell includes CMOS decoding logic, a CMOS-toECL converter, an ECL flip-flop and a bipolar differential pair to steer the current. The ECL flip-flop is clocked by a global ECL differential clock, and the differential output of the flip-flop directly steers the bipolar differential pair. The measured static nonlinearities INL and DNL were both approximately $2 \mathrm{LSB}$. The measured SFDR was $43 d B$ at a generated frequency of approximately $0.3 \cdot f_{s} \quad\left(f_{s}=\right.$ $100 \mathrm{MSamples} / \mathrm{s}$ ) and $50 \mathrm{~dB}$ at approximately $1 / 10 \cdot f_{s}$.

\section{ACKNOWLEDGEMENTS}

The authors thank their colleagues at the ASIC group at SINTEF Instrumentation, especially Geir Førre, for helpful and inspiring discussions. Furthermore, the authors thank Mustapha Slimane Kadi for valuable help and Jan Frode Lønnum at SINTEF DELAB for assistance during testing. This work has been carried out under contract of EU under ESPRIT contract 8795 (AMFIS). SINTEF's work has been funded by the Research Council of Norway. Ivan H. H. Jørgensen acknowledges the doctorate studentship granted by the Danish Research Council.

\section{REFERENCES}

[1] Geir Førre, Svein Anders Tunheim, Jakob Gakkestad and Arne Kjensmo, "Design, Simulation and Layout of a 100 Msamples/s CMOS DAC", SINTEF Report no. STF31 F93034, September 1993.

[2] Ivan Harald Holger J $\varnothing$ gensen, "BiCMOS Current Cell with Cascoded CMOS Current Sink", Internal SINTEF Memo, November 1994.

[3] S.Chin and C.Wu, "A 10-b 125MHz CMOS Digital-to-Analog Converter (DAC) with Threshold-Voltage Compensated Current Sources", IEEE JSSC, vol. 29, no. 11, November 1994.

[4] B. G. Henriques and José E. Franca, "A High-Speed Programmable CMOS Interface System Combining D/A Conversion and FIR filtering", IEEE JSSC, vol. 29, no. 8, August 1994.

[5] T.Wu et. al, "A Low Glitch 10-bit 75-MHz CMOS Video D/A Converter", IEEE JSSC, vol. 30, no. 1, January 1995.

[6] Y.Nakamura et al., "A 10-b 70MS/s CMOS D/A-converter", IEEE JSSC vol. 26, no. 4, pp. 637-642, Apr. 1991.

[7] C.Bastiaansen et al., "A 10-b 40-MHz 0.8 $\mu \mathrm{m}$ CMOS Current-Output D/A-converter", IEEE JSSC, vol. 26, no. 7, July 1991.

[8] Sverre Dale Moen and Svein Anders Tunheim, "High Level Simulations of a 100Msamples/s DAC", SINTEF Report no. STF31 F94044, October 1994. 